\title{
Homogeneous Learning: Self-Attention Decentralized Deep Learning
}

This paper was downloaded from TechRxiv (https://www.techrxiv.org).

\section{LICENSE}

CC BY 4.0

SUBMISSION DATE / POSTED DATE

$24-12-2021$ / 28-12-2021

CITATION

Sun, Yuwei; Ochiai, Hideya (2021): Homogeneous Learning: Self-Attention Decentralized Deep Learning. TechRxiv. Preprint. https://doi.org/10.36227/techrxiv.17456786.v1

DOI

10.36227/techrxiv.17456786.v1 


\title{
Homogeneous Learning: Self-Attention Decentralized Deep Learning
}

\author{
YUWEI SUN'1,2, HIDEYA OCHIAI', (Member, IEEE) \\ ${ }^{1}$ Graduate School of Information Science and Technology, University of Tokyo, Tokyo 1138654 Japan (e-mail: ywsun@ g.ecc.u-tokyo.ac.jp \& \\ ochiai@elab.ic.i.u-tokyo.ac.jp) \\ ${ }^{2}$ RIKEN AIP, Tokyo 1030027 Japan \\ Corresponding author: Yuwei Sun (e-mail: ywsun@g.ecc.u-tokyo.ac.jp).
}

This work was partly supported by the JRA program at RIKEN AIP.

\begin{abstract}
Federated learning (FL) has been facilitating privacy-preserving deep learning in many walks of life such as medical image classification, network intrusion detection, and so forth. Whereas it necessitates a central parameter server for model aggregation, which brings about delayed model communication and vulnerability to adversarial attacks. A fully decentralized architecture like Swarm Learning allows peer-to-peer communication among distributed nodes, without the central server. One of the most challenging issues in decentralized deep learning is that data owned by each node are usually nonindependent and identically distributed (non-IID), causing time-consuming convergence of model training. To this end, we propose a decentralized learning model called Homogeneous Learning (HL) for tackling non-IID data with a self-attention mechanism. In HL, training performs on each round's selected node, and the trained model of a node is sent to the next selected node at the end of each round. Notably, for the selection, the self-attention mechanism leverages reinforcement learning to observe a node's inner state and its surrounding environment's state, and find out which node should be selected to optimize the training. We evaluate our method with various scenarios for two different image classification tasks. The result suggests that HL can achieve a better performance compared with standalone learning and greatly reduce both the total training rounds by $50.8 \%$ and the communication cost by $74.6 \%$ for decentralized learning with nonIID data.
\end{abstract}

INDEX TERMS Collective intelligence, Distributed computing, Knowledge transfer, Multi-layer neural network, Supervised learning

\section{INTRODUCTION}

Centralized deep learning in high performance computing (HPC) environments has been facilitating the advancement in various areas such as drug discovery, disease diagnosis, cybersecurity, and so on. Despite its broad applications in many walks of life, the associated potential data exposure of training sources and privacy regulation violation have greatly decreased the practicality of such centralized learning architecture. In particular, with the promotion of GDPR [1], data collection for centralized model training has become more and more difficult.

Decentralized deep learning (DDL) is a concept to bring together distributed data sources and computing resources while taking the full advantage of deep learning models. Nowadays, DDL such as Federated Learning (FL) [2] has been offering promising solutions to social issues of data privacy, especially in large-scale multi-agent learning. These massively distributed nodes can facilitate diverse use cases, such as industrial IoT [3], environment monitoring with smart sensors [4], human behavior recognition with surveillance cameras [5], connected autonomous vehicles control [6], [7], network intrusion detection [8], [9], and so forth.

Though FL has been attracting great attention due to the privacy-preserving architecture, recent years' upticks in adversarial attacks cause its hardly guaranteed trustworthiness. FL encounters various threats, such as backdoor attacks [10][12], information stealing attacks [13], and so on. On the contrast, fully decentralized architectures like Swarm Learning (SL) [14] leverages the blockchain, smart contract, and other state-of-the-art decentralization technologies to offer a more practical solution. Whereas, a great challenge of it has been deteriorated performance in model training with nonindependent identically distributed (non-IID) data, leading to extremely increased time of model convergence. 


\section{A. OUR CONTRIBUTIONS}

We propose a self-attention decentralized deep learning model called Homogeneous Learning (HL). HL leverages a shared communication policy for adaptive model sharing among nodes. A starter node initiates a training task and by iteratively sending the trained model and performing training on each round's selected node its model is updated for achieving the training goal. Notably, a node selection decision is made by reinforcement learning agents based on the current selected node's inner state and outer state of its surrounding environment to maximize a reward for moving towards the training goal. Finally, comprehensive experiments and evaluation results suggest that HL can accelerate the model training on non-IID data with $50.8 \%$ fewer training rounds and reduce the communication cost by $74.6 \%$.

\section{B. PAPER OUTLINE}

This paper is organized as follows. Section III demonstrates the most recent work about DDL and methodologies for tackling data heterogeneity problems in model training. Section III discusses assumptions and definitions used in this research. Section IV presents the technical underpinnings of Homogeneous Learning, including the local machine learning (ML) task model, the reinforcement learning model, and the self-attention mechanism to learn an optimized communication policy. Section $\nabla$ demonstrates experimental evaluations for tackling various image classification tasks with three baseline models applied. Section VI concludes the paper and gives out a future direction of this work.

\section{RELATED WORK}

\section{A. DECENTRALIZED DEEP LEARNING}

In recent years, lots of DDL architectures have been proposed leveraging decentralization technologies such as the blockchain and ad hoc networks. For instance, $\mathrm{Li}$ et al. [15] presented a blockchain-based decentralized learning framework based on the FISCO blockchain system. They applied the architecture to train AlexNet models on the FEMNIST dataset. Similarly, Lu et al. [16] demonstrated a blockchain empowered secure data sharing architecture for FL in industrial IoT. Furthermore, Mowla et al. [17] proposed a client group prioritization technique leveraging the Dempster-Shafer theory for unmanned aerial vehicles (UAVs) in flying ad-hoc networks. HL is a fully decentralized machine learning model sharing architecture based on decentralization technology such as token exchanges.

\section{B. CONVERGENCE OPTIMIZATION WITH SKEWED DATA}

In a real-life application, usually data owned by different clients in such a decentralized system are skewed. For this reason, the model training is slow and even diverges. Methodologies for tackling such data heterogeneity such as FL, have been studied for a long time. For example, Sener et al. [18] presented the K-Center clustering algorithm which aims to find a representative subset of data from a very large collection such that the performance of the model based on the small subset and that based on the whole collection will be as close as possible. Moreover, Wang et al. [19] demonstrated reinforcement learning-based client selection in FL, which counterbalances the bias introduced by non-IID data thus speeding up the global model's convergence. Sun et al. [8] proposed the Segmented-FL to tackle heterogeneity in massively distributed network intrusion traffic data, where clients with highly skewed training data are dynamically divided into different groups for model aggregation respectively at each round. Furthermore, Zhao et al. [20] presented a data-sharing strategy in FL by creating a small data subset globally shared between all the clients. Likewise, Jeong et al. [21] proposed the federated augmentation where each client augments its local training data using a generative neural network. Different from the aforementioned approaches, HL leverages a self-attention mechanism that optimizes the communication policy in DDL using reinforcement learning models. It is aimed to reduces computational and communication cost of decentralized training on skewed data.

\section{PRELIMINARIES}

\section{A. CLASSIFICATION TASK}

We specifically consider supervised learning with $C$ categories in the entire dataset $D$. Let $x \in \mathbb{R}^{D}$ be a sample and $y \in\{1,2, \ldots, C\}=Y$ a label. $D$ consists of a collection of $N$ samples as $D=\left\{\left(x_{i}, y_{i}\right)\right\}_{i=1}^{N}$. Suppose that $f$ denotes a neural network classifier taking an input $x_{i}$ and outputting a $C$-dimensional real-valued vector where the $j$ th element of the output vector represents the probability that $x_{i}$ is recognized as class $j$. Given $f(x)$, the prediction is given by $\hat{y}=\arg \max _{j} f(x)_{j}$ where $f(x)_{j}$ denotes the $j$ th element of $f(x)$. The training of neural network is attained by minimizing the following loss function with respect to the model parameter $\theta$

$$
J(\theta, D)=\frac{1}{N} \sum_{i=1}^{N} \ell\left(y_{i}, f\left(x_{i} ; \theta\right)\right) .
$$

\section{B. DECENTRALIZED DEEP LEARNING}

We assume there are $K$ clients. The $k$ th client has its own dataset $D^{(k)}:=\left\{\left(x_{i}, y_{i}\right)\right\}_{i=1}^{N^{(k)}}$ where $N^{(k)}$ is the sample size of dataset $D^{(k)}$. Here, $\cup_{i=1}^{K} D^{(k)}=D$ and $N=\sum_{k=1}^{K} N^{(k)}$. We also suppose that each client cannot share data each other due to some reason, mainly due to data confidentiality. Decentralized deep learning (DDL) is a framework to obtain a global model that is trained over the entire data without sharing distributed samples. For instance, federated learning (FL) [2] consists of the parameter server (PS) and lots of clients. Let $G_{t}$ be the global model of the PS and $L_{t}^{(k)}$ be the local model of the client $k$ at the round $t$. For each training round $t$, a subset of clients $K_{\text {selected }}$ is selected for model training with the latest global model parameters $G_{t}$ based on their own dataset $D^{\left(k \in K_{\text {selected }}\right)}$. Then, the trained models $L_{t+1}^{\left(k \in K_{\text {selected }}\right)}$ are sent back to the PS for aggregation thus improving the joint global model $G_{t+1}$. 
Moreover, a peer-to-peer DDL system consists of distributed nodes functioning as both the server and the client based on decentralization technologies such as blockchain [14]-[16], token-exchange [17], and so on. For example, the token-exchange validates and issues security tokens to enable nodes to obtain appropriate access credentials for exchanging resources without the central server. This is different from FL where the parameter server plays the key role in learning process control of model sharing.

\section{DATA HETEROGENEITY}

The challenges related to heterogeneity of nodes in DDL refer to two categories, i.e., data heterogeneity and hardware heterogeneity. Notably, data heterogeneity results in timeconsuming convergence or divergence of model learning. Let $p(x \mid y)$ be the common data distribution of the entire data $D$. We assume the common distribution $p(x \mid y)$ is shared by all nodes. Then, Node $k$ has $p_{k}(y)$. We first consider an independent and identically distributed (IID) setting, i.e., $p_{i}(x, y)=p(x \mid y) p_{i}(y)$ s.t. $p_{i}(y)=p_{j}(y)$ for all $i \neq j$. Under this assumption, the data distribution of the entire dataset can be represented by a node's local data distribution. Unfortunately, in real-life application, samples held by clients are usually skewed with various data distributions, i.e., $p_{i}(x, y)=p(x \mid y) p_{i}(y)$ s.t. $p_{i}(y) \neq p_{j}(y)$ for all $i \neq j$. Node 1 follows $p 1(x, y)$ and Node 2 follows $p 2(x, y)$. We further define and clarify such data heterogeneity as follows: given samples $\left\{\left(x_{i}, y_{i}\right)\right\}_{i=1}^{N^{(k)}}$ in node $k$ 's local dataset $D^{(k)}$, when $\alpha$ samples are from a single main data class $c^{(k)}$ subject to $\alpha>\frac{N^{(k)}}{C}$ and the remaining samples are randomly drawn from the other $C-1$ data classes, the heterogeneity level $H^{(k)}$ of node $k$ is formulated as $H^{(k)}=-p\left(y_{i}=c^{(k)}\right) *$ $\log \left(p\left(y_{i} \neq c^{(k)}\right)\right)$ subject to $y_{i} \in\left\{y_{i}\right\}_{i=1}^{N^{(k)}}$. Moreover, we assign a main data class $c^{(k)}=k \% C$ to node $k$.

\section{COMMUNICATION OVERHEAD}

Communication overhead in DDL usually refers to the payload of shared local model parameters [22], [23] and communication distances between nodes that share a model with each other. We mainly discuss the later case here. In particular, let $d_{i, j}$ be the communication distance from node $i$ to node $j$. Dis $s_{i \times j}$ is a symmetrical matrix where the bidirectional distances between two nodes are equal and the distance to a node itself $d_{i, j \mid i=j}$ is zero. In addition, each distance $d_{i, j \mid i \neq j}$ in the matrix is a random numerical value taken between 0 and $\beta$, where $\beta$ denotes the upper bound of the relative distance (Equation 2).

$$
\begin{gathered}
\text { Dis } s_{i \times j}=\left(\begin{array}{cccc}
d_{1,1} & d_{1,2} & \cdots & d_{1, j} \\
d_{2,1} & d_{2,2} & \cdots & d_{2, j} \\
\vdots & \vdots & \ddots & \vdots \\
d_{i, 1} & d_{i, 2} & \cdots & d_{i, j}
\end{array}\right) \\
\text { subject to }: d_{i, j \mid i=j}=0, d_{i, j}=d_{j, i}, \\
d_{i, j \mid i \neq j} \in(0, \beta]
\end{gathered}
$$

\section{HOMOGENEOUS LEARNING}

We propose a novel decentralized deep learning architecture called Homogeneous Learning (HL) (Fig. 1). HL leverages reinforcement learning (RL) agents to learn a shared communication policy of node selection, thus contributing to fast convergence of model training and reducing communication cost as well. In HL, each node has two machine learning (ML) models, i.e., a local ML task model $L^{(k)}$ for the multiclassification task and an RL model $L^{D Q N}$ for the node selection in peer-to-peer communications.

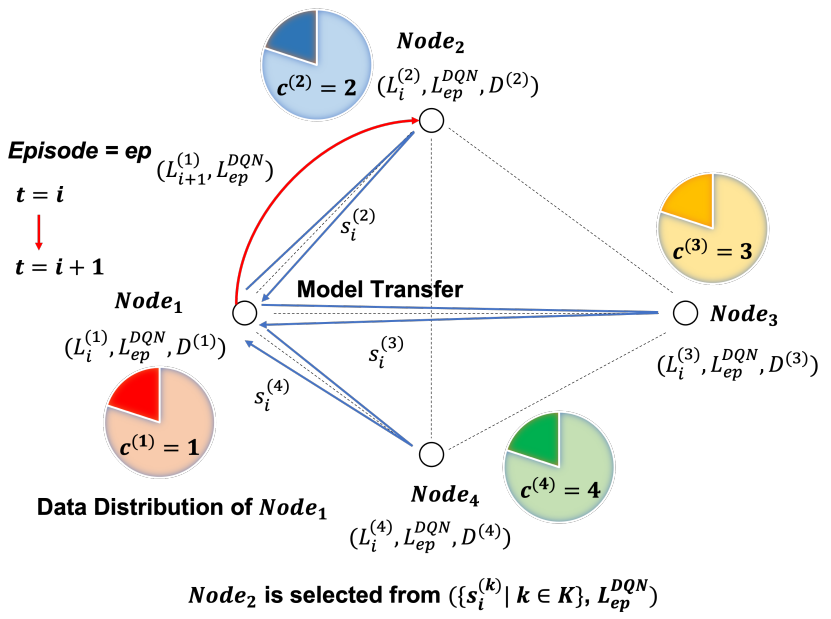

FIGURE 1: Homogeneous learning: self-attention decentralized deep learning.

\section{A. LOCAL ML TASK MODEL}

We assume the $K$ nodes in HL share the same model architecture for a classification task, which we call a local ML task model. Let $y_{i}$ be the layeri's output of $L^{(k)} \cdot y_{i}=$ $f_{i}\left(W_{i} y_{i-1}\right), i=1, \ldots, p, y_{0}=x$, where $f_{i}$ is the activation function, $W_{i}$ is the weight matrix of layer $i, y_{i-1}$ represents the output of the previous layer, and $p$ is the number of layers in $L^{(k)}$. Notably, we employ a three-layer convolutional neural network (CNN) with an architecture as follows: the first convolutional layer of the $\mathrm{CNN}$ model has a convolution kernel of size $5 \times 5$ with a stride of 1 and it takes one input plane and it produces 20 output planes, followed by a ReLU activation function; the second convolutional layer takes 20 input planes and produces 50 output planes and it has a convolution kernel of size $5 \times 5$ with a stride of 1 , followed by ReLU; the output is flattened followed by a linear transformation of a fully connected layer, which takes as input the tensor and outputs a tensor of size $C$ representing the $C$ categories. Moreover, the categorical cross-entropy is employed to compute a loss $J\left(L_{t}^{(k)}, D^{(k)}\right)$. After that, we apply as a learning function the Adam to update the model.

\section{B. REINFORCEMENT LEARNING MODEL}

In addition to the local ML task model, each node $k$ in $\mathrm{HL}$ is also associated with a reinforcement learning (RL) 
model $L^{D Q N}$. The goal of the RL model is to learn a communication policy for the node selection in decentralized learning. There are three main components of the RL model, the state $s$, the action $a$, and the reward $r$. Based on the input state $s$, the RL model outputs an action $a$ for the next node selection, and at the same time, updates itself by correlating the attained reward $r$ with the performed action $a$. As a result, the recursive self-improvement of the RL model allows a node to constantly explore the relation between the system's performance and the selection policy (i.e., the self-attention mechanism in HL), contributing to faster convergence of model learning.

Every round $t$, a $\mathrm{RL}$ model observes the state $s_{t}$ from two different sources, i.e., model parameters $s_{t}^{(k)}$ of the selected node $k$ and parameters of models in the surrounding environment $\left\{s_{t}^{(i)} \mid i \in K, i \neq k\right\}$. In particular, we employ a deep Q-network (DQN), which approximates a state-value function in a Q-learning framework with a neural network. Let $y_{i}^{D Q N}$ be the layeri's output of $L^{D Q N}$. $y_{i}^{D Q N}=f_{i}^{D Q N}\left(W_{i}^{D Q N} y_{i-1}^{D Q N}\right), i=1, \ldots, q, y_{0}^{D Q N}=s$, where $f_{i}^{D Q N}$ is the activation function of layeri, $W_{i}^{D Q N}$ is the weight matrix of layer $i, y_{i-1}^{D Q N}$ represents the output of the previous layer, and $q$ is the number of layers in $L^{D Q N}$. Notably, a DQN model consisting of three fully connected layers is applied (Fig. 2). The two hidden layers consist of 500 and 200 neurons respectively, using as an activation function the ReLU. The output layer with a linear activation function consists of $K$ neurons that output the rewards for selecting each node $k$ respectively, $k \in\{1,2, \ldots, K\}$. Furthermore, at each round $t$, the node with the largest reward will be selected. $\hat{a_{t}}=\arg \max _{j} f^{D Q N}\left(s_{t}\right)_{j}$. Consequently, the RL model selects and sends the trained local model $L_{t+1}^{(k)}$ of node $k$ to the next node $a_{t}$. As such, the local ML task model of node $a_{t}$ is then updated to $L_{t+1}^{(k)}$.

To understand the training of the RL model, we first define the input state $s_{t}$. The state $s_{t}$ is a concatenated vector of the flattened model parameters of all nodes in the systems. $s_{t}=$ $\left\{s_{t}^{(k)} \mid k \in K\right\}$. To efficiently represent the state and compute the RL model prediction, we adopt the principal component analysis (PCA) to reduce the dimension of the state $s_{t}$ from an extremely large number (e.g., 33580 dimensions for the model parameters used in an MNIST classification task with an input size of $28 \times 28$ ) to $K$, where $K$ is the number of nodes. $K$ is adopted due to the minimum possible dimension of a PCA-based output vector is the number of input samples. Then, we define the output reward $r_{t}$. Every round $t$, a trained ML task model is evaluated on a hold-out validation set $D_{v a l}$, and the reward $r_{t}$ can be computed from the validation accuracy $V a l A c c_{t}$, the communication distance between the current node $k$ and the next selected node $a_{t}$, and a penalty of minus one for taking each training step. $r_{t}=$ $32^{\left(\text {ValAcc }_{t}-\text { GoalAcc }\right)}-d_{a_{t-1}, a_{t}}-1$, where Goal Acc denotes the desired performance on the validation set and $d_{a_{t-1}, a_{t}}$ is the communication distance drawn from the distance matrix $D i s_{i \times j}$. We employ an exponentially increasing function $32^{(\cdot)}$ to distinguish between different validation results when the ML task model is close to convergence when only small variance is observed in the results. In addition, an episode reward $R$ is the accumulative reward of the current reward and discounted future rewards in the whole training process of HL. $R=\sum_{t=1}^{T} \gamma^{t-1} r_{t}$, where $T$ is the total training rounds of $\mathrm{HL}$ in one episode.

With DQN, we often use experience replay during training. A RL model's experience at each time step $t$ is stored in a data set called the replay memory. Let $e_{t}$ be the model's experience at time $t$. $e_{t}=\left(s_{t}, a_{t}, r_{t}, s_{t+1}\right)$, where $r_{t}$ is the reward given the current state-action pair $\left(s_{t}, a_{t}\right)$ and $s_{t+1}$ is the state of the ML task models after training. We assume a finite size limit $M$ of the replay memory, and it will only store the last $M$ experiences. Moreover, to facilitate constant exploration of a RL model, epsilon is a factor to control the probability of the next node being selected by the RL model. In particular, for each round, a random numerical value between 0 and 1 is obtained and compared with the current epsilon value Epsilon $_{e p}$ where $e p$ denotes the current episode. Then if the randomly picked value is greater than Epsilon $_{e p}$, the next node will be selected by the RL model. Otherwise, a random action of node selection will be performed. For either case, an experience sample $e_{t}=\left(s_{t}, a_{t}, r_{t}, s_{t+1}\right)$ will be stored in the replay memory. The decentralized learning terminates when either the model achieves the desired performance on the validation set or exceeds a maximum number of rounds $T_{\max }$ the learning progress of which is called an episode of HL. For each episode, we apply the epsilon decay $\rho$ to gradually increase the possibility of the RL model's decision-making. Epsilon $_{e p+1}=$ Epsilon $_{e p} \cdot e^{-\rho}$, where Epsilon Ep $+1_{\text {is }}$ is the computed epsilon for the next episode and $e$ is the Euler's number that is approximately equal to 2.718 .

Furthermore, at the end of each episode $e p$, the RL model is trained on a small subset $b$ of samples randomly drawn from the replay memory. We adopt as a learning function the Adam. Then, the optimization of the DQN model is formulated in (3). The updated DQN model is shared with the next selected node. As such, the RL model performs better and better in predicting the expected rewards of selecting each node for the next round, which results in the increase of the episode reward $R$ by selecting the node with the largest expected reward at each round $t$.

$$
\begin{aligned}
& r_{t+1}=\max _{a_{i}} f^{D Q N}\left(s_{i+1} ; L_{e p}^{D Q N}\right)_{a_{i}} \\
& \hat{r_{t}}=f^{D Q N}\left(s_{i} ; L_{e p}^{D Q N}\right) a_{i} \\
& Q\left(L_{e p}^{D Q N}\right)=\sum_{i=1}^{B} \ell\left(r_{t}+\gamma \hat{r}_{t+1}, \hat{r_{t}}\right) \\
& \theta^{*}=\arg \min _{\theta} Q(\theta), \text { subject to } \theta=L_{e p}^{D Q N}
\end{aligned}
$$

Where $a_{i}$ denotes the predicted next step's action that maximizes the future reward, $\gamma$ denotes the discount factor 


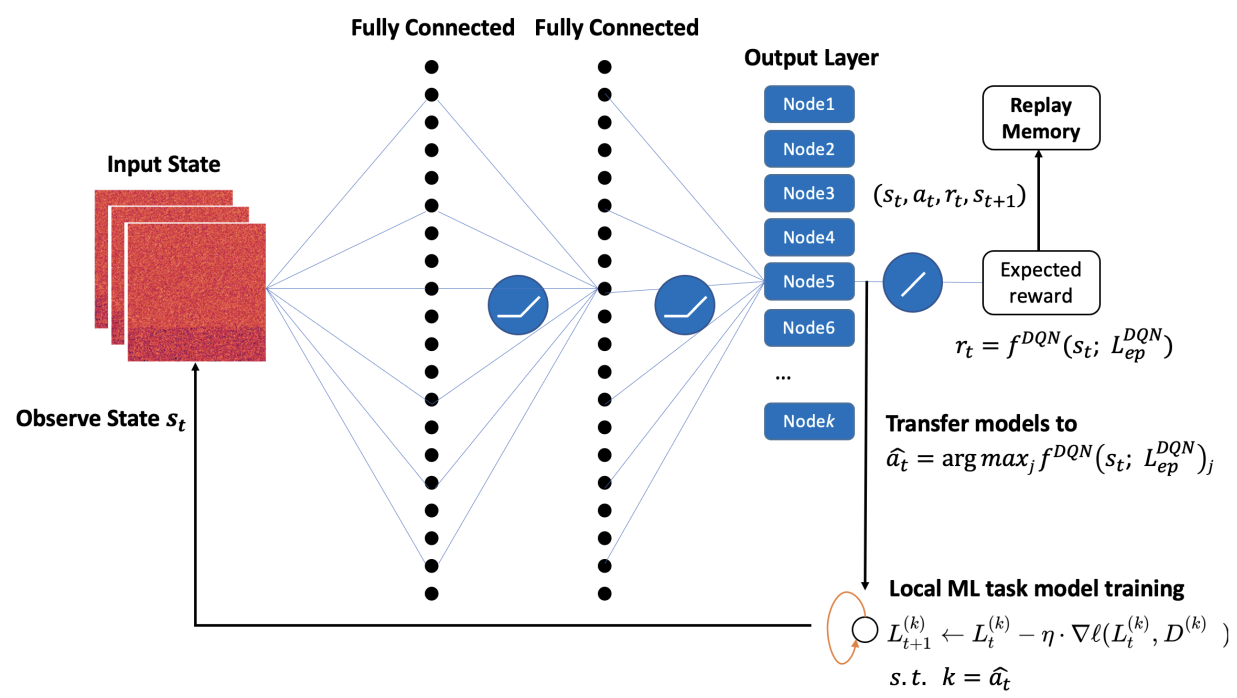

FIGURE 2: Next-node selection based on the RL model of HL.

of the future reward, $B$ denotes the number of samples in the subset $b$, and $Q$ is the mean squared error loss function.

Finally, the model training of HL is formulated as Algorithm 1. Algorithm 2 demonstrates the application phase of HL after obtaining the optimized communication policy of node selection.

\section{EXPERIMENTS}

\section{A. SETTINGS}

We evaluated the proposed method based on two different image classification tasks of MNIST and Fashion-MNIST. MNIST [24] is a handwritten digit image dataset containing 50,000 training samples and 10,000 test samples labeled as $0-9$, and Fashion-MNIST [25] is an image collection of 10 types of clothing containing 50,000 training samples and 10,000 test samples labeled as shoes, t-shirts, dresses, and so on. The image data in these two datasets are grayscale with a size of $28 \times 28$. Moreover, we considered both a 10-node scenario and a 100-node scenario of HL for tackling the two classification tasks respectively. The machine learning library we used to build the system is Tensorflow. All experiments were conducted on a GPU server with 60 AMD Ryzen Threadripper CPUs, two NVidia Titan RTX GPUs with 24 GB RAM each, and Ubuntu 18.04.5 LTS OS.

To compare the performance, we adopted three different baseline models, which are a centralized learning model based on the data collection of all nodes, a decentralized learning model based on a random communication policy, and a standalone learning model based on a node's local data without communication. For each type of model, we used the same architecture of the ML task model and the same training hyperparameters. We assigned the training goal of a model validation accuracy of 0.80 for the MNIST classification task and 0.70 for the Fashion-MNIST classification task respectively, using the hold-out test set in the corresponding dataset.

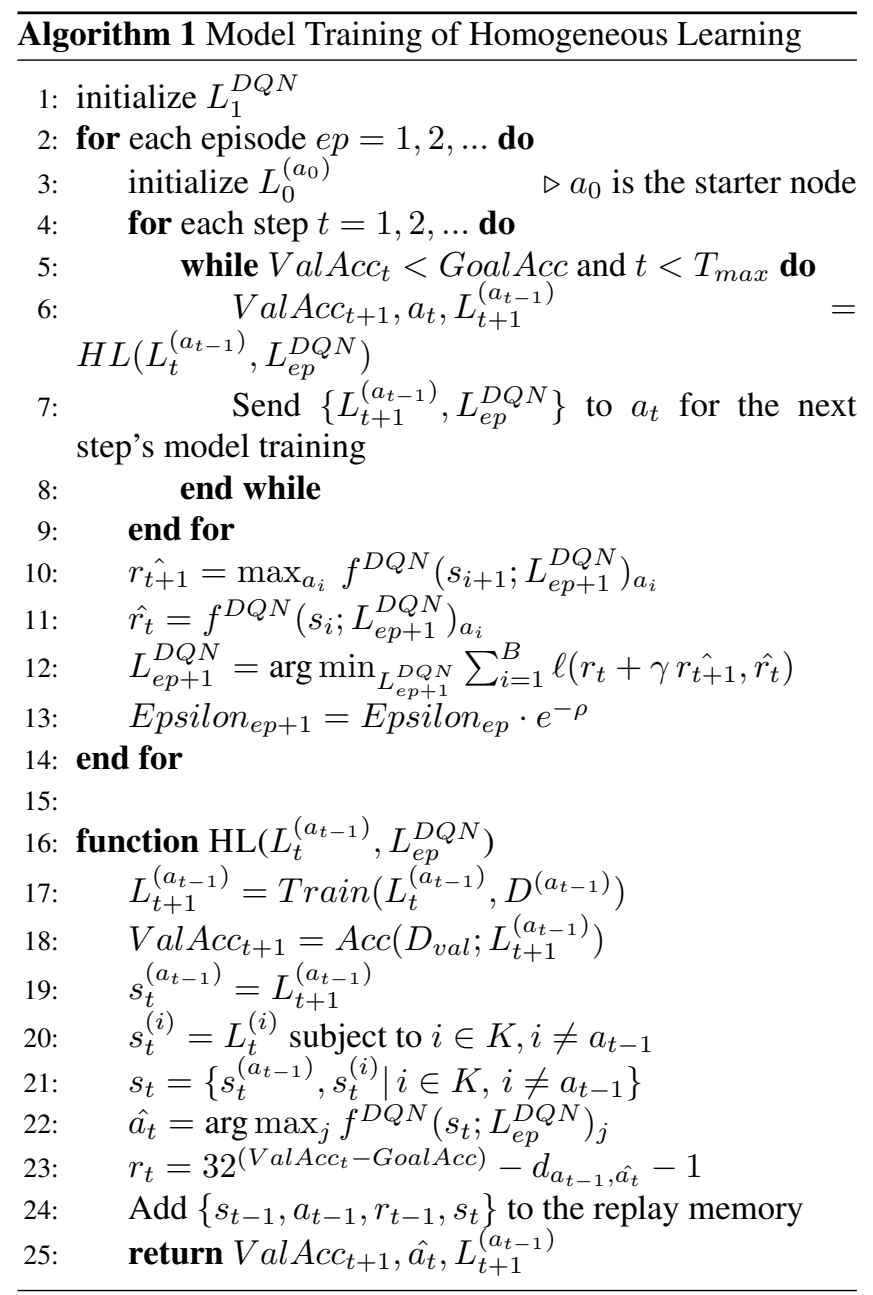




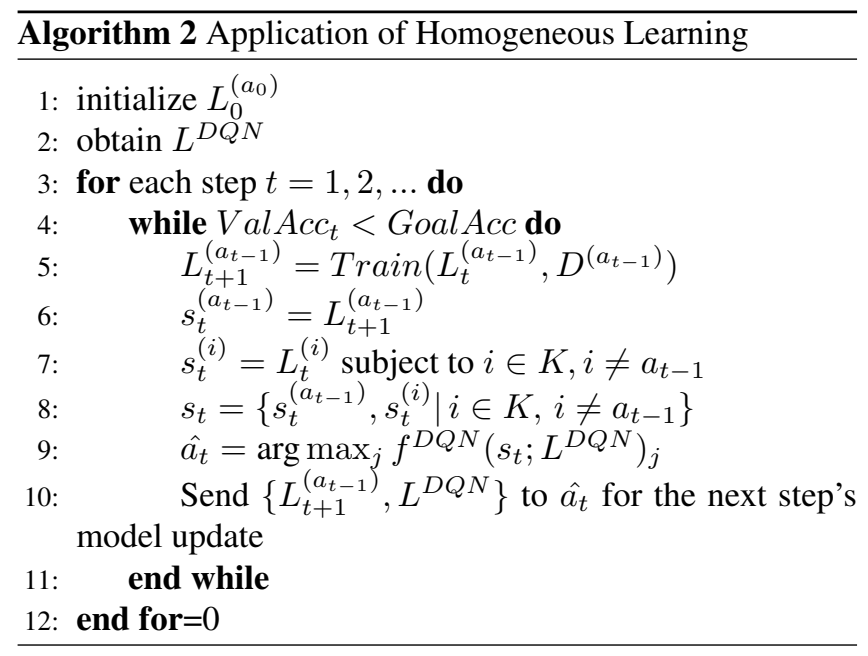

In addition, for the standalone learning, we adopted the early stopping to monitor the validation loss of the model at each epoch with a patience of five, which automatically terminated the training process when there appeared no further decrease in the validation loss of the model for the last five epochs. In both the centralized learning and the standalone learning, evaluation was performed at the end of each training epoch. On the other hand, in the two decentralized learning cases, due to multiple models existing in the system, evaluation was performed on the trained local model of each step's selected node with the same hold-out test set above.

Furthermore, for the decentralized learning, each node $k$ owned a total of 500 skewed local training data that have a heterogeneity level $H=0.56\left(p\left(y_{i}=c^{(k)}\right)=0.8\right)$ subject to $y_{i} \in\left\{y_{i}\right\}_{i=1}^{N^{(k)}}$. The discussion on various heterogeneity levels is in Section V-B3 In HL, to generate the distance matrix, the relative communication cost represented by the distance between two different nodes $d_{i, j \mid i \neq j}$ takes a random numerical value between 0 and 0.1 . A random seed of 0 was adopted for the reproducibility of the distance matrix (See Appendix A). For the local ML task model training, we adopted an epoch of one with a batch size of 32. A further discussion on the selection of these two hyperparameters can be found in Appendix B. The Adam was applied as an optimization function with a learning rate of 0.001 .

\section{B. EXPERIMENTAL RESULTS}

1) Common Communication Policy Learning

As aforementioned, each node $k$ has a specific main data class $c^{(k)}$. We considered a starter node that had a main data class of digit ' 0 ' for MNIST and a main class of T-shirt for Fashion-MNIST. Then, starting from the starter node, a local ML task model was trained on the current node's local data and sent to the next step's node decided by either the RL model or a random action every step, depending on the epsilon of the current episode (we adopted an initial epsilon of one and a decay rate of 0.02). For each episode, we applied a maximum step of 35 for MNIST and 100 for Fashion-MNIST. Moreover, the ML task model and the RL

TABLE 1: Hyperparameters in Homogeneous Learning

ML TASK MODEL

\begin{tabular}{|c|c|c|c|}
\hline Epoch & 1 & Episode & 120 \\
\hline Batch size & 32 & Future reward & 0.9 \\
\hline Learning rate & 0.001 & discount & \\
\hline Optimization & Adam & Epsilon decay & 0.02 \\
\hline function & & Epoch & 1 \\
\hline Maximum step & 35 (MNIST)/ & Batch size & 16 \\
\hline & 100 (Fashion-MNIST) & Learning rate & 0.001 \\
\hline
\end{tabular}

model were updated using the hyperparameters in Table. 1 . In addition, we applied a maximum replay memory size of 50,000 and a minimum size of 128 , where the training of the DQN model started only when there were more than 128 samples in the replay memory and the oldest samples would be removed when samples were more than the maximum capacity.

For each episode, we computed the step rewards and the episode reward for the model training to achieve the desired performance. With the advancement of episodes, the communication policy evolved to improve the episode reward thus benefiting better decision-making of the next-node selection. Fig. 3 illustrates the episode reward and the mean reward over the last 10 episodes of HL in the 10-node and 100-node scenarios for MNIST and Fashion-MNIST respectively.

\section{2) Computational and Communication Cost}

Computational cost refers to the required total rounds for a system to achieve the desired performance and was evaluated for all methods. Communication cost refers to the total communication distance of model sharing from the starter node to the last selected node and was evaluated for the two decentralized learning methods. Notably, to evaluate the computational and communication cost, we conducted 10 individual experiments using different random seeds for each method and adopted as final results the best cases of node selection over the last five episodes when the learned communication policy was prone to settling. The experiments were performed in the 10-node scenario for the MNIST task.

As shown in Fig. 5]a, due to limited local training data, the standalone learning appeared to be extremely slow after the validation accuracy reached 0.70 . It terminated with a final accuracy of around 0.75 with the early-stopping strategy. Moreover, by comparing the decentralized learning methods with and without the self-attention mechanism, the result suggests that our proposed method of HL can greatly reduce the total training rounds facilitating the model convergence. In addition, though centralized learning shows the fastest convergence, it suffers from problems of data privacy.

As shown in Fig. 5.b, the bottom and top of the error bars represent the $25_{t h}$ and $75_{t h}$ percentiles respectively, the line inside the box shows the median value, and outliers are shown as open circles. As a result, it shows that HL can greatly reduce the total training rounds by $50.8 \%$ and the communication cost by $74.6 \%$ in decentralized learning of 

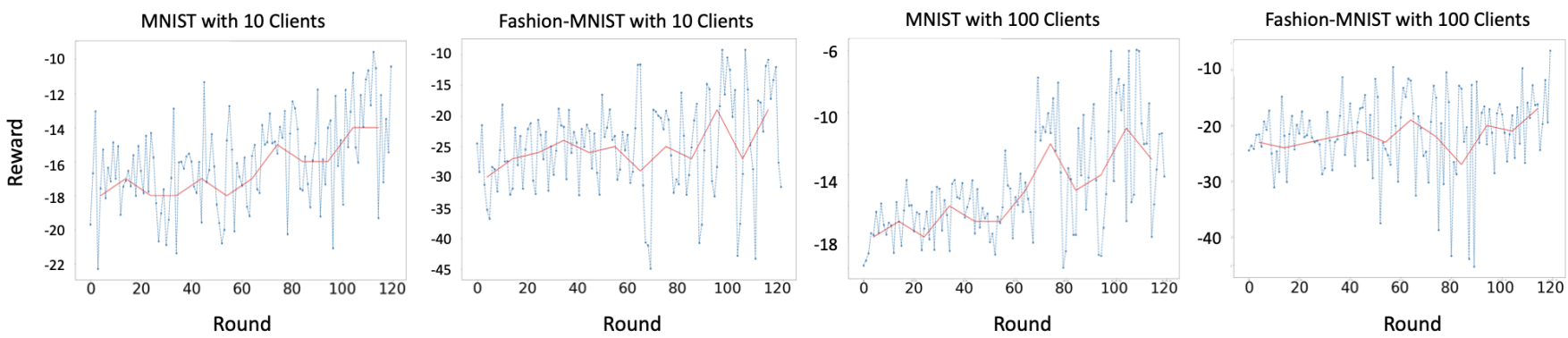

FIGURE 3: With the increase of training episodes, the mean reward over last 10 episodes is gradually increasing. The DQN model learned a better communication policy by training on samples from the replay memory, contributing to a faster convergence of model training.

the 10-node scenario for the MNIST task.

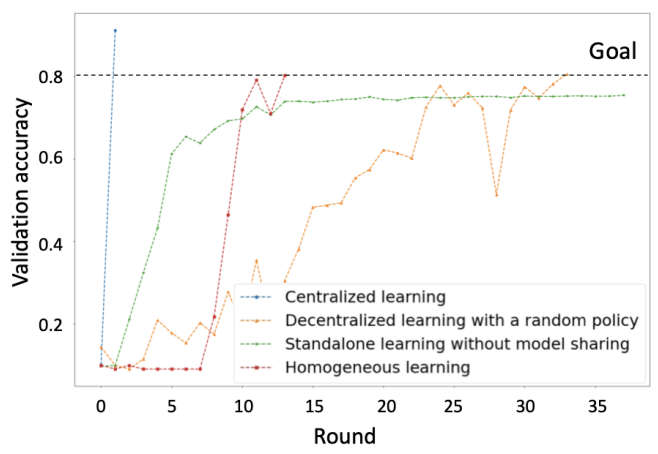

(a)

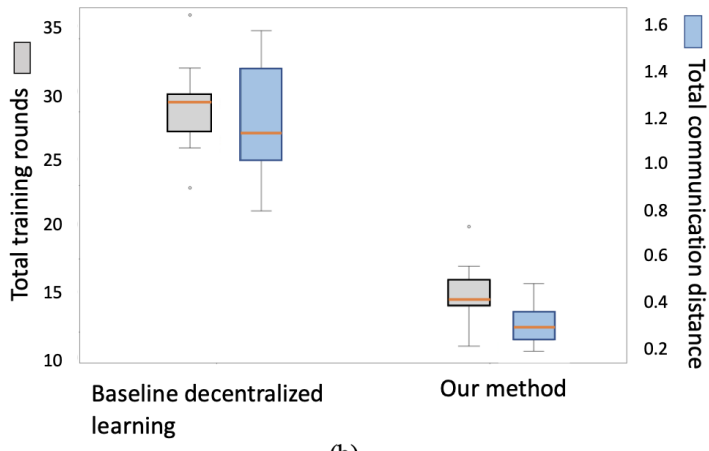

(b)

FIGURE 4: (a) Total training rounds based on different methods. (b) Cost comparison between the random policybased decentralized learning and our method HL. Each error bar illustrates 10 individual experiments' results.

\section{3) HL with Various Heterogeneity Levels}

We further studied the performance of the proposed method with different heterogeneity levels $H=\{0.24,0.56,0.90\}$ $\left(p\left(y_{i}=c^{(k)}\right)=\{0.6,0.8,0.9\}\right.$ subject to $\left.y_{i} \in\left\{y_{i}\right\}_{i=1}^{N^{(k)}}\right)$. We evaluated the model performance in the 10-node scenario for the MNIST task. For the cases of $H=\{0.24,0.56\}$, we applied a maximum training step of 35 as defined above. For the case of $H=0.90$, we applied a maximum training step of 80 instead due to a challenging convergence of the ML task model using the highly skewed local training data. Fig. 5 illustrates the comparison of computational cost between $\mathrm{HL}$ and the random policy-based decentralized learning.

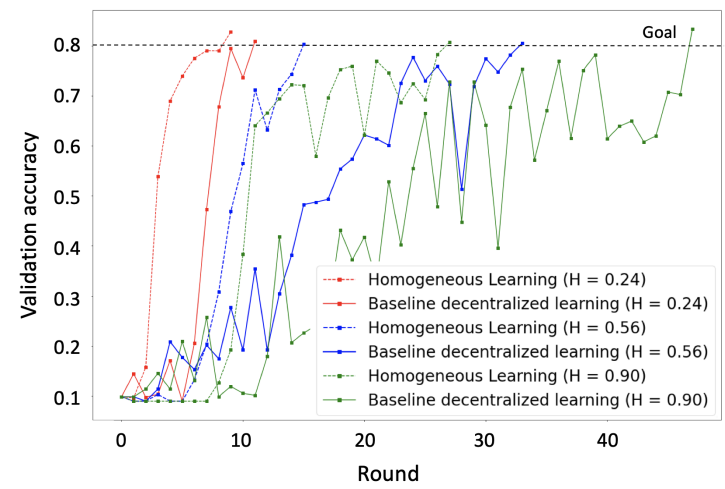

FIGURE 5: Total training rounds when applying local training data with various heterogeneity levels. The dash lines are the results of HL and the solid lines are the results of the random policy-based decentralized learning. Different colors represent different heterogeneity levels $H=$ $\{0.24,0.56,0.90\}$. As we can see, HL becomes more efficient when training on distributed data with a higher heterogeneity level, contributing to a larger ratio of reduced total training rounds.

\section{CONCLUSION}

Decentralized deep learning (DDL) leveraging distributed data sources contributes to a better neural network model while safeguarding data privacy. Despite the broad applications of DDL models such as federated learning and swarming learning, the challenges regarding edge heterogeneity especially the data heterogeneity have greatly limited their scalability. In this research, we proposed a self-attention decentralized deep learning method of Homogeneous Learning (HL) that recursively updates a shared communication policy by observing the system's state and the gained reward for taking an action based on the observation. We comprehensively evaluated the proposed method in the 10-node and 100-node scenarios for tackling two different image classification tasks, applying as criteria the computational and communication 
cost. The evaluation results show that HL can greatly reduce the training cost with highly skewed distributed data. In future, a decentralized learning model that can leverage various communication policies in parallel is considered for the further study of HL.

\section{APPENDIX A COMMUNICATION DISTANCE MATRIX}

Fig. 6 illustrates the generated distance matrix $D_{i \times j}$ in the 10 -node scenario when applying a $\beta$ of 0.1 and a random seed of 0 .

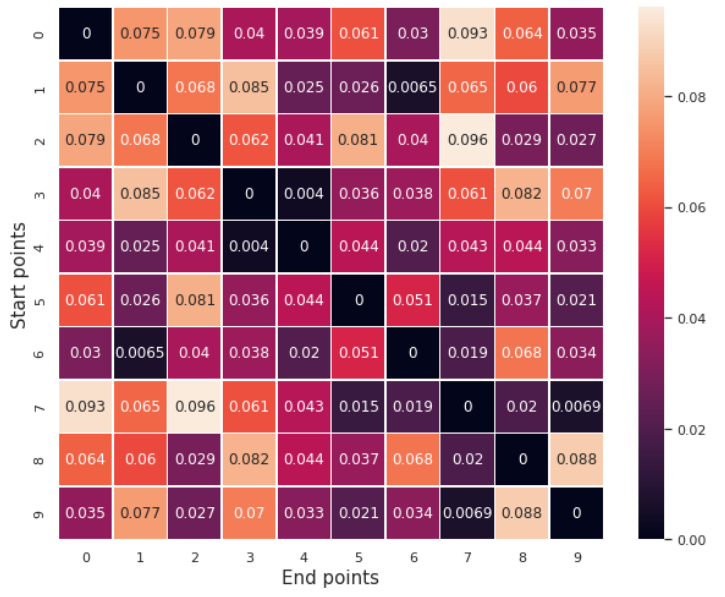

FIGURE 6: The distance matrix $D_{i \times j}$ in the 10-node scenario.

\section{APPENDIX B MODEL DISTRIBUTION REPRESENTATION OPTIMIZATION}

Under the assumption of data heterogeneity, to allow a reinforcement learning (RL) agent to efficiently learn a communication policy by observing model states in the systems, a trade-off between the batch size and the epoch of local foundation model training was discussed. Fig. 7 illustrates the trained models' weights distribution in the 10-node scenario after applying the principal component analysis (PCA), with different batch sizes and epochs applied to train on the MNIST dataset. Moreover, it shows the 100-node scenario where each color represents nodes with the same main data class. As shown in the graphs, various combinations of these two parameters have different distribution representation capabilities. By comparing the distribution density and scale, we found that when adopting a batch size of 32 and an epoch of one the models distribution was best represented, which could facilitate the policy learning of an agent.

\section{REFERENCES}

[1] General data protection regulation. Accessed: 2021-09-22.

[2] Jakub Konečný, H. Brendan McMahan, Felix X. Yu, Peter Richtarik, Ananda Theertha Suresh, and Dave Bacon. Federated learning: Strategies for improving communication efficiency. 2016.
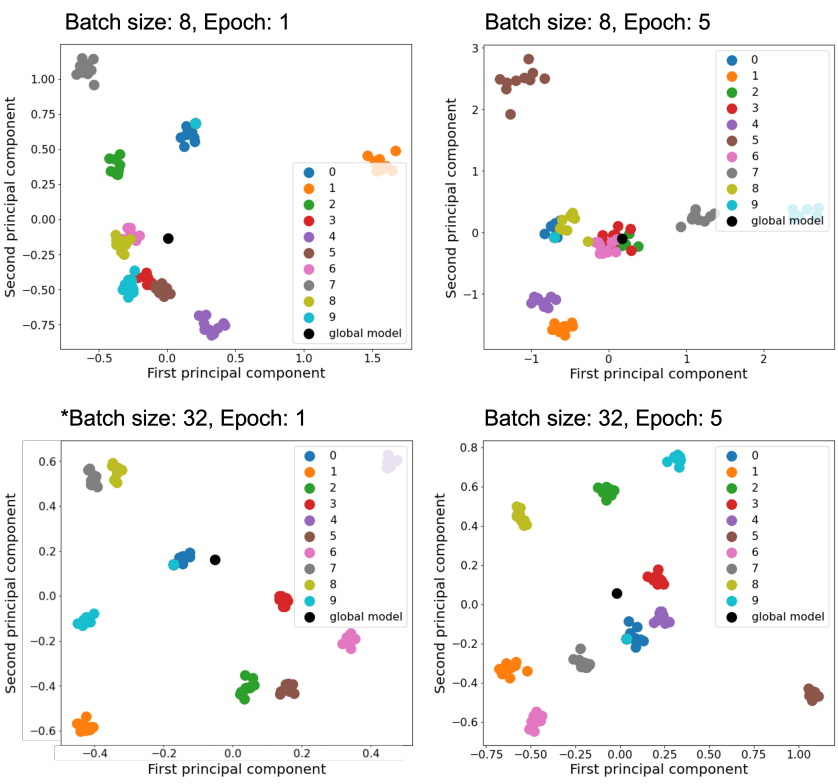

Batch size: 32, Epoch: 5

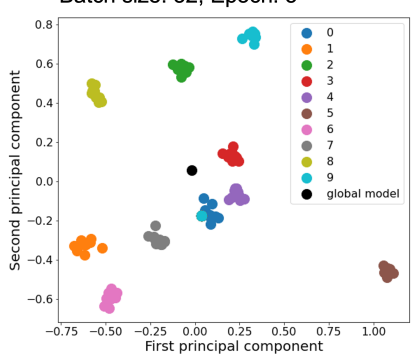

FIGURE 7: Model distribution representation optimization.

[3] Mani Parimala, R. M. Swarna Priya, Quoc-Viet Pham, Kapal Dev, Praveen Kumar Reddy Maddikunta, Thippa Reddy Gadekallu, and Thien HuynhThe. Fusion of federated learning and industrial internet of things: A survey. ArXiv, abs/2101.00798, 2021.

[4] Yujia Gao, Liang Liu, Binxuan Hu, Tianzi Lei, and Huadong Ma. Federated region-learning for environment sensing in edge computing system. IEEE Transactions on Network Science and Engineering, 7(4):2192-2204, 2020.

[5] Yang Liu, Anbu Huang, Yun Luo, He Huang, Youzhi Liu, Yuanyuan Chen, Lican Feng, Tianjian Chen, Han Yu, and Qiang Yang. Fedvision: An online visual object detection platform powered by federated learning. Proceedings of the AAAI Conference on Artificial Intelligence, 34(08):1317213179, Apr. 2020.

[6] Shiva Raj Pokhrel and Jinho Choi. Federated learning with blockchain for autonomous vehicles: Analysis and design challenges. IEEE Transactions on Communications, 68(8):4734-4746, 2020.

[7] Boyi Liu, Lujia Wang, Ming Liu, and Chengzhong Xu. Lifelong federated reinforcement learning: A learning architecture for navigation in cloud robotic systems. CoRR, abs/1901.06455, 2019.

[8] Yuwei Sun, Hiroshi Esaki, and Hideya Ochiai. Adaptive intrusion detection in the networking of large-scale lans with segmented federated learning. IEEE Open J. Commun. Soc., 2:102-112, 2021.

[9] Sawsan Abdul Rahman, Hanine Tout, Chamseddine Talhi, and Azzam Mourad. Internet of things intrusion detection: Centralized, on-device, or federated learning? IEEE Network, 34(6):310-317, 2020.

[10] H. Brendan McMahan, Daniel Ramage, Kunal Talwar, and Li Zhang. Learning differentially private recurrent language models. 2018.

[11] Di Cao, Shan Chang, Zhijian Lin, Guohua Liu, and Donghong Sun. Understanding distributed poisoning attack in federated learning. pages 233-239, 2019.

[12] T. Nguyen, P. Rieger, Markus Miettinen, and A. Sadeghi. Poisoning attacks on federated learning-based iot intrusion detection system. 2020.

[13] Moming Duan, Duo Liu, Xianzhang Chen, Renping Liu, Yujuan Tan, and Liang Liang. Self-balancing federated learning with global imbalanced data in mobile systems. IEEE Trans. Parallel Distributed Syst., 32(1):5971, 2021.

[14] Stefanie Warnat-Herresthal, Hartmut Schultze, Krishnaprasad Lingadahalli Shastry, Sathyanarayanan Manamohan, Saikat Mukherjee, Vishesh Garg, Ravi Sarveswara, Kristian Händler, Peter Pickkers, N. Ahmad Aziz, Sofia Ktena, Christian Siever, Michael Kraut, Milind Desai, Bruno Monnet, Maria Saridaki, Charles Martin Siegel, Anna Drews, Melanie NueschGermano, Heidi Theis, Mihai G. Netea, Fabian Theis, Anna C. Aschenbrenner, Thomas Ulas, Monique M.B. Breteler, Evangelos J. GiamarellosBourboulis, Matthijs Kox, Matthias Becker, Sorin Cheran, Michael S. 
Woodacre, Eng Lim Goh, Joachim L. Schultze, and German COVID19 OMICS Initiative (DeCOI). Swarm learning for decentralized and confidential clinical machine learning. Nature 594, 265-270, 2021.

[15] Yuzheng Li, Chuan Chen, Nan Liu, Huawei Huang, Zibin Zheng, and Qiang Yan. A blockchain-based decentralized federated learning framework with committee consensus. IEEE Netw., 35(1):234-241, 2021.

[16] Yunlong Lu, Xiaohong Huang, Yueyue Dai, Sabita Maharjan, and Yan Zhang. Blockchain and federated learning for privacy-preserved data sharing in industrial iot. IEEE Transactions on Industrial Informatics, 16(6):4177-4186, 2020.

[17] Nishat I. Mowla, Nguyen H. Tran, Inshil Doh, and Kijoon Chae. Federated learning-based cognitive detection of jamming attack in flying ad-hoc network. IEEE Access, 8:4338-4350, 2020.

[18] Ozan Sener and Silvio Savarese. Active learning for convolutional neural networks: A core-set approach. 2018.

[19] Hao Wang, Zakhary Kaplan, Di Niu, and Baochun Li. Optimizing federated learning on non-iid data with reinforcement learning. pages 1698-1707, 2020.

[20] Yue Zhao, Meng Li, Liangzhen Lai, Naveen Suda, Damon Civin, and Vikas Chandra. Federated learning with non-iid data. CoRR abs/1806.00582, 2018.

[21] Eunjeong Jeong, Seungeun Oh, Hyesung Kim, Jihong Park, Mehdi Bennis and Seong-Lyun Kim. Communication-efficient on-device machine learning: Federated distillation and augmentation under non-iid private data. CoRR, abs/1811.11479, 2018.

[22] Chaoyang He, Murali Annavaram, and Salman Avestimehr. Group knowledge transfer: Federated learning of large cnns at the edge. 2020.

[23] Abhishek Singh, Praneeth Vepakomma, Otkrist Gupta, and Ramesh Raskar. Detailed comparison of communication efficiency of split learning and federated learning. CoRR, abs/1909.09145, 2019.

[24] Yann LeCun, Corinna Cortes, and CJ Burges. Mnist handwritten digit database. ATT Labs [Online]. Available: http://yann.lecun.com/exdb/mnist, 2, 2010.

[25] Han Xiao, Kashif Rasul, and Roland Vollgraf. Fashion-mnist: a novel image dataset for benchmarking machine learning algorithms. CoRR, abs/1708.07747, 2017.

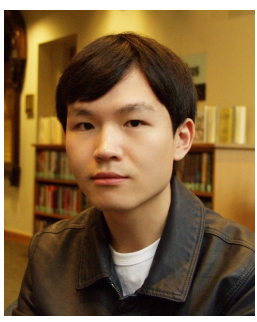

YUWEI SUN (M'20) is a Ph.D.'s student in the Graduate School of Information Science and Technology at the University of Tokyo. He received B.E. in Computer Science and Technology in 2018 from North China Electric Power University and M.E. in Information and Communication Engineering with honors in 2021 from the University of Tokyo. In 2020, he was the fellow of the Advanced Study Program (ASP) at the Massachusetts Institute of Technology. He has been working with the Campus Computing Centre at the United Nations University Centre on Cybersecurity since 2019. He is a member of the AI Security and Privacy Team at the RIKEN Center for Advanced Intelligence Project working on trustworthy AI, and a research fellow at Japan Society for the Promotion of Science (JSPS).

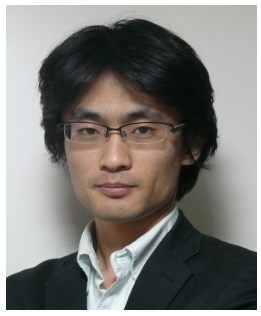

HIDEYA OCHIAI (M'10) is an associate professor of the University of Tokyo, Japan. He received B.E. in 2006, M.E. in 2008, and Ph.D. in 2011 from the same university. His research interests have been sensor networking, delay tolerant networking, and building automation systems, IoT protocols, and cyber-security. $\mathrm{He}$ is involved in the standardization of facility information access protocol in IEEE1888, ISO/IEC, and ASHRAE. 